\title{
General fractional integral inequalities for convex and $m$-convex functions via an extended generalized Mittag-Leffler function
}

\author{
G. Farid ${ }^{1}$, K.A. Khan'², N. Latif ${ }^{3}$, A.U. Rehman' and S. Mehmood ${ }^{4 *}$
}

"Correspondence:

smjg227@gmail.com

${ }^{4}$ GBPS Sherani, Hazro Attock

Pakistan

Full list of author information is

available at the end of the article

\begin{abstract}
In this paper some new general fractional integral inequalities for convex and $m$-convex functions by involving an extended Mittag-Leffler function are presented. These results produce inequalities for several kinds of fractional integral operators. Some interesting special cases of our main results are also pointed out.
\end{abstract}

MSC: 26A51; 26A33; 33E12

Keywords: Convex function; $m$-convex function; Mittag-Leffler function; Generalized fractional integral operators; Hadamard inequality

\section{Introduction, definitions, and preliminaries}

Convex functions are very important in the field of integral inequalities. A lot of fractional integral inequalities and novel results have been established due to convex functions (for more details, see $[1,8,13,14])$.

Definition 1 A function $f: I \rightarrow \mathbb{R}$, where $I$ is an interval in $\mathbb{R}$, is said to be a convex function if

$$
f(t x+(1-t) y) \leq t f(x)+(1-t) f(y)
$$

holds for $t \in[0,1]$ and $x, y \in I$.

A convex function $f: I \rightarrow \mathbb{R}$ is also equivalently defined by the Hadamard inequality

$$
f\left(\frac{a+b}{2}\right) \leq \frac{1}{b-a} \int_{a}^{b} f(x) d x \leq \frac{f(a)+f(b)}{2}
$$

where $a, b \in I, a<b$.

The concept of $m$-convexity was introduced in [17] and since then many properties, especially inequalities, have been obtained for this class of functions (see $[3,6,7,18]$ ).

(c) The Author(s) 2018. This article is distributed under the terms of the Creative Commons Attribution 4.0 International License (http://creativecommons.org/licenses/by/4.0/), which permits unrestricted use, distribution, and reproduction in any medium, provided you give appropriate credit to the original author(s) and the source, provide a link to the Creative Commons license, and indicate if changes were made. 
Definition 2 A function $f:[0, b] \rightarrow \mathbb{R}, b>0$ is called $m$-convex, where $m \in[0,1]$, if for every $x, y \in[0, b]$ and $t \in[0,1]$, we have

$$
f(t x+m(1-t) y) \leq t f(x)+m(1-t) f(y) .
$$

For $m=1$, we recapture the definition of convex functions, and for $m=0$, the definition of star-shaped functions defined on $[0, b]$. We recall that a function $f:[0, b] \rightarrow \mathbb{R}$ is called star-shaped if

$$
f(t x) \leq t f(x) \quad \text { for all } t \in[0,1] \text { and } x \in[0, b]
$$

If we denote by $K_{m}(b)$ the set of $m$-convex functions defined on $[0, b]$ for which $f(0)<0$, then

$$
K_{1}(b) \subset K_{m}(b) \subset K_{0}(b),
$$

whenever $m \in(0,1)$. Note that in the class $K_{1}(b)$ there are only convex functions $f$ : $[0, b] \rightarrow \mathbb{R}$ for which $f(0) \leq 0$ (see [4]), while $k_{0}(b)$ contains star-shaped functions.

Example $1.1([6])$ The function $f:[0, \infty) \rightarrow \mathbb{R}$, given by

$$
f(x)=\frac{1}{12}\left(x^{4}-5 x^{3}+9 x^{2}-5 x\right)
$$

is a $\frac{16}{17}$-convex function but it is not $m$-convex for any $m \in\left(\frac{16}{17}, 1\right]$.

For more results and inequalities related to $m$-convex functions, one can consult, for example, $[3,6,7]$ along with the references therein.

Recently in [2] Andrić et al. defined an extended generalized Mittag-Leffler function $E_{\mu, \alpha, l}^{\gamma, \delta, k, c}(\cdot ; p)$ as follows.

Definition 3 ([2]) Let $\mu, \alpha, l, \gamma, c \in \mathbb{C}, \Re(\mu), \Re(\alpha), \Re(l)>0, \Re(c)>\Re(\gamma)>0$ with $p \geq 0, \delta>$ 0 , and $0<k \leq \delta+\Re(\mu)$. Then the extended generalized Mittag-Leffler function $E_{\mu, \alpha, l}^{\gamma, \delta, k, c}(t ; p)$ is defined by

$$
E_{\mu, \alpha, l}^{\gamma, \delta, c, c}(t ; p)=\sum_{n=0}^{\infty} \frac{\beta_{p}(\gamma+n k, c-\gamma)}{\beta(\gamma, c-\gamma)} \frac{(c)_{n k}}{\Gamma(\mu n+\alpha)} \frac{t^{n}}{(l)_{n \delta}}
$$

where $\beta_{p}$ is the generalized beta function defined by

$$
\beta_{p}(x, y)=\int_{0}^{1} t^{x-1}(1-t)^{y-1} e^{-\frac{p}{t(1-t)}} d t
$$

and $(c)_{n k}$ is the Pochhammer symbol defined as $(c)_{n k}=\frac{\Gamma(c+n k)}{\Gamma(c)}$.

In [2] properties of the generalized Mittag-Leffler function are discussed, and it is given that $E_{\mu, \alpha, l}^{\gamma, \delta, k, c}(t ; p)$ is absolutely convergent for $k<\delta+\Re(\mu)$. Let $S$ be the sum of series of absolute terms of the Mittag-Leffler function $E_{\mu, \alpha, l}^{\gamma, \delta, k, c}(t ; p)$, then we have $\left|E_{\mu, \alpha, l}^{\gamma, \delta, c, c}(t ; p)\right| \leq S$. We use this property of Mittag-Leffler function in our results where we need. 
The corresponding left and right sided extended generalized fractional integral operators are defined as follows.

Definition 4 ([2]) Let $\omega, \mu, \alpha, l, \gamma, c \in \mathbb{C}, \Re(\mu), \Re(\alpha), \Re(l)>0, \Re(c)>\Re(\gamma)>0$ with $p \geq 0$, $\delta>0$ and $0<k \leq \delta+\Re(\mu)$. Let $f \in L_{1}[a, b]$ and $x \in[a, b]$. Then the extended generalized fractional integral operators $\epsilon_{\mu, \alpha, l, \omega, a^{+}}^{\gamma, \delta, k, c} f$ and $\epsilon_{\mu, \alpha, l, \omega, b^{-}}^{\gamma, \delta, k} f$ are defined by

$$
\left(\epsilon_{\mu, \alpha, l, \omega, a^{+}}^{\gamma, \delta, k, c} f\right)(x ; p)=\int_{a}^{x}(x-t)^{\alpha-1} E_{\mu, \alpha, l}^{\gamma, \delta, k, c}\left(\omega(x-t)^{\mu} ; p\right) f(t) d t
$$

and

$$
\left(\epsilon_{\mu, \alpha, l, \omega, b^{-}}^{\gamma, \delta, k, c} f\right)(x ; p)=\int_{x}^{b}(t-x)^{\alpha-1} E_{\mu, \alpha, l}^{\gamma, \delta, k, c}\left(\omega(t-x)^{\mu} ; p\right) f(t) d t
$$

From extended generalized fractional integral operators, we have

$$
\begin{aligned}
\left(\epsilon_{\mu, \alpha, l, \omega, a^{+}}^{\gamma, \delta, k} 1\right)(x ; p) \\
\quad=\int_{a}^{x}(x-t)^{\alpha-1} E_{\mu, \alpha, l}^{\gamma, \delta, k, c}\left(w(x-t)^{\mu} ; p\right) d t \\
\quad=\int_{a}^{x}(x-t)^{\alpha-1} \sum_{n=0}^{\infty} \frac{\mathrm{B}_{p}(\gamma+n k, c-\gamma)}{\mathrm{B}(\gamma, c-\gamma)} \frac{(c)_{n k}}{\Gamma(\mu n+\alpha)} \frac{w^{n}(x-t)^{\mu n}}{(l)_{n \delta}} d t \\
=\sum_{n=0}^{\infty} \frac{\mathrm{B}_{p}(\gamma+n k, c-\gamma)}{\mathrm{B}(\gamma, c-\gamma)} \frac{(c)_{n k}}{\Gamma(\mu n+\alpha)} \frac{w^{n}}{(l)_{n \delta}} \int_{a}^{x}(x-t)^{\mu n+\alpha-1} d t \\
=(x-a)^{\alpha} \sum_{n=0}^{\infty} \frac{\mathrm{B}_{p}(\gamma+n k, c-\gamma)}{\mathrm{B}(\gamma, c-\gamma)} \frac{(c)_{n k}}{\Gamma(\mu n+\alpha)} \frac{w^{n}}{(l)_{n \delta}}(x-a)^{\mu n} \frac{1}{\mu n+\alpha} .
\end{aligned}
$$

Hence

$$
\left(\epsilon_{\mu, \alpha, l, \omega, a^{+}}^{\gamma, \delta, k, c} 1\right)(x ; p)=(x-a)^{\alpha} E_{\mu, \alpha+1, l}^{\gamma, \delta, k, c}\left(w(x-a)^{\mu} ; p\right),
$$

and similarly

$$
\left(\epsilon_{\mu, \alpha, l, \omega, b^{-}}^{\gamma, \delta, k, c} 1\right)(x ; p)=(b-x)^{\alpha} E_{\mu, \alpha+1, l}^{\gamma, \delta, k, c}\left(w(b-x)^{\mu} ; p\right) .
$$

We use the following notations in our results:

$$
C_{\alpha, a^{+}}(x ; p)=\left(\epsilon_{\mu, \alpha, l, \omega, a^{+}}^{\gamma, \delta, k, c} 1\right)(x ; p)
$$

and

$$
C_{\alpha, b^{-}}(x ; p)=\left(\epsilon_{\mu, \alpha, l, \omega, b^{-}}^{\gamma, \delta, k, c} 1\right)(x ; p)
$$

For more information related to Mittag-Leffler functions and corresponding fractional integral operators, the readers are referred to $[9-12,15,16,19]$. 
In this paper we give general fractional integral inequalities for convex and $m$-convex functions by involving an extended Mittag-Leffler function and deduce some results already published in $[1,5,6,8,13]$. Also we give a Hadamard type inequality for convex and $m$-convex functions by involving an extended Mittag-Leffler function.

\section{Main results}

Here we give some fractional integral inequalities for convex and $m$-convex functions via an extended generalized Mittag-Leffler function and corresponding fractional integral operators given in (3) and (4). The following lemma is useful to establish the results.

Lemma 2.1 Let $f:[a, m b] \rightarrow \mathbb{R}$ be a differentiable function such that $f^{\prime} \in L_{1}[a, m b]$ with $0 \leq a<m b$. Also let $g:[a, m b] \rightarrow \mathbb{R}$ be a continuous function on $[a, m b]$, then the following identity for extended generalized fractional integral operators holds:

$$
\begin{aligned}
& \left(\int_{a}^{m b} g(s) E_{\mu, \alpha, l}^{\gamma, \delta, c,}\left(\omega s^{\mu} ; p\right) d s\right)^{\alpha}[f(a)+f(m b)] \\
& \quad-\alpha \int_{a}^{m b}\left(\int_{a}^{t} g(s) E_{\mu, \alpha, l}^{\gamma, \delta, k, c}\left(\omega s^{\mu} ; p\right) d s\right)^{\alpha-1} g(t) E_{\mu, \alpha, l}^{\gamma, \delta, k}\left(\omega t^{\mu} ; p\right) f(t) d t \\
& \quad-\alpha \int_{a}^{m b}\left(\int_{t}^{m b} g(s) E_{\mu, \alpha, l}^{\gamma, \delta, k, c}\left(\omega s^{\mu} ; p\right) d s\right)^{\alpha-1} g(t) E_{\mu, \alpha, l}^{\gamma, \delta, k, c}\left(\omega t^{\mu} ; p\right) f(t) d t \\
& =\int_{a}^{m b}\left(\int_{a}^{t} g(s) E_{\mu, \alpha, l}^{\gamma, \delta, k}\left(\omega s^{\mu} ; p\right) d s\right)^{\alpha} f^{\prime}(t) d t \\
& \quad-\int_{a}^{m b}\left(\int_{t}^{m b} g(s) E_{\mu, \alpha, l}^{\gamma, \delta, k, c}\left(\omega s^{\mu} ; p\right) d s\right)^{\alpha} f^{\prime}(t) d t .
\end{aligned}
$$

Proof On integrating by parts one can have

$$
\begin{aligned}
\int_{a}^{m b} & \left(\int_{a}^{t} g(s) E_{\mu, \alpha, l}^{\gamma, \delta, k, c}\left(\omega s^{\mu} ; p\right) d s\right)^{\alpha} f^{\prime}(t) d t \\
= & \left(\int_{a}^{m b} g(s) E_{\mu, \alpha, l}^{\gamma, \delta, k, c}\left(\omega s^{\mu} ; p\right) d s\right)^{\alpha} f(m b) \\
& -\alpha \int_{a}^{m b}\left(\int_{a}^{t} g(s) E_{\mu, \alpha, l}^{\gamma, \delta, k, c}\left(\omega s^{\mu} ; p\right) d s\right)^{\alpha-1} g(t) E_{\mu, \alpha, l}^{\gamma, \delta, k, c}\left(\omega t^{\mu} ; p\right) f(t) d t
\end{aligned}
$$

and

$$
\begin{aligned}
\int_{a}^{m b} & \left(\int_{t}^{m b} g(s) E_{\mu, \alpha, l}^{\gamma, \delta, k, c}\left(\omega s^{\mu} ; p\right) d s\right)^{\alpha} f^{\prime}(t) d t \\
= & -\left(\int_{a}^{m b} g(s) E_{\mu, \alpha, l}^{\gamma, \delta, k, c}\left(\omega s^{\mu} ; p\right) d s\right)^{\alpha} f(a) \\
& +\alpha \int_{a}^{m b}\left(\int_{t}^{m b} g(s) E_{\mu, \alpha, l}^{\gamma, \delta, k, c}\left(\omega s^{\mu} ; p\right) d s\right)^{\alpha-1} g(t) E_{\mu, \alpha, l}^{\gamma, \delta, k, c}\left(\omega t^{\mu} ; p\right) f(t) d t .
\end{aligned}
$$

Subtracting (9) from (8), we get (7) which is the required identity.

If we take $m=1$ in (7), then we get the following identity for a convex function. 
Corollary 2.2 Let $f:[a, b] \subseteq[0, \infty) \rightarrow \mathbb{R}$ be a differentiable function such that $f^{\prime} \in L_{1}[a, b]$ with $a<b$. Also let $g:[a, b] \rightarrow \mathbb{R}$ be continuous on $[a, b]$, then the following identity for extended generalized fractional integral operators holds:

$$
\begin{aligned}
& \left(\int_{a}^{b} g(s) E_{\mu, \alpha, l}^{\gamma, \delta, k, c}\left(\omega s^{\mu} ; p\right) d s\right)^{\alpha}[f(a)+f(b)] \\
& \quad-\alpha \int_{a}^{b}\left(\int_{a}^{t} g(s) E_{\mu, \alpha, l}^{\gamma, \delta, k, c}\left(\omega s^{\mu} ; p\right) d s\right)^{\alpha-1} g(t) E_{\mu, \alpha, l}^{\gamma, \delta, k, c}\left(\omega t^{\mu} ; p\right) f(t) d t \\
& \quad-\alpha \int_{a}^{b}\left(\int_{t}^{b} g(s) E_{\mu, \alpha, l}^{\gamma, \delta, k, c}\left(\omega s^{\mu} ; p\right) d s\right)^{\alpha-1} g(t) E_{\mu, \alpha, l}^{\gamma, \delta, k, c}\left(\omega t^{\mu} ; p\right) f(t) d t \\
& =\int_{a}^{b}\left(\int_{a}^{t} g(s) E_{\mu, \alpha, l}^{\gamma, \delta, k, c}\left(\omega s^{\mu} ; p\right) d s\right)^{\alpha} f^{\prime}(t) d t \\
& \quad-\int_{a}^{b}\left(\int_{t}^{b} g(s) E_{\mu, \alpha, l}^{\gamma, \delta, k, c}\left(\omega s^{\mu} ; p\right) d s\right)^{\alpha} f^{\prime}(t) d t .
\end{aligned}
$$

We use identity (7) to establish the following fractional integral inequality.

Theorem 2.3 Let $f:[a, m b] \rightarrow \mathbb{R}$ be a differentiable function such that $f^{\prime} \in L_{1}[a, m b]$ with $0 \leq a<m b$. Also let $g:[a, m b] \rightarrow \mathbb{R}$ be a continuous function on $[a, m b]$. If $\left|f^{\prime}\right|$ is an $m$ convex function on $[a, m b]$, then the following inequality for extended generalized fractional integral operators holds:

$$
\begin{aligned}
& \mid\left(\int_{a}^{m b} g(s) E_{\mu, \alpha, l}^{\gamma, \delta, k, c}\left(\omega s^{\mu} ; p\right) d s\right)^{\alpha}(f(a)+f(m b)) \\
& \quad-\alpha \int_{a}^{m b}\left(\int_{a}^{t} g(s) E_{\mu, \alpha, l}^{\gamma, \delta, k, c}\left(\omega s^{\mu} ; p\right) d s\right)^{\alpha-1} g(t) E_{\mu, \alpha, l}^{\gamma, \delta, k, c}\left(\omega t^{\mu} ; p\right) f(t) d t \\
& \quad-\alpha \int_{a}^{m b}\left(\int_{t}^{m b} g(s) E_{\mu, \alpha, l}^{\gamma, \delta, k, c}\left(\omega s^{\mu} ; p\right) d s\right)^{\alpha-1} g(t) E_{\mu, \alpha, l}^{\gamma, \delta, k, c}\left(\omega t^{\mu} ; p\right) f(t) d t \mid \\
& \quad \leq \frac{(m b-a)^{\alpha+1}\|g\|_{\infty}^{\alpha} S^{\alpha}}{(\alpha+1)}\left(\left|f^{\prime}(a)\right|+m\left|f^{\prime}(b)\right|\right)
\end{aligned}
$$

for $k<\delta+\Re(\mu)$ and $\|g\|_{\infty}=\sup _{t \in[a, m b]}|g(t)|$.

Proof From Lemma 2.1, we have

$$
\begin{aligned}
& \mid\left(\int_{a}^{m b} g(s) E_{\mu, \alpha, l}^{\gamma, \delta, k, c}\left(\omega s^{\mu} ; p\right) d s\right)^{\alpha}(f(a)+f(m b)) \\
& \quad-\alpha \int_{a}^{m b}\left(\int_{a}^{t} g(s) E_{\mu, \alpha, l}^{\gamma, \delta, k, c}\left(\omega s^{\mu} ; p\right) d s\right)^{\alpha-1} g(t) E_{\mu, \alpha, l}^{\gamma, \delta, k, c}\left(\omega t^{\mu} ; p\right) f(t) d t \\
& \quad-\alpha \int_{a}^{m b}\left(\int_{t}^{m b} g(s) E_{\mu, \alpha, l}^{\gamma, \delta, k, c}\left(\omega s^{\mu} ; p\right) d s\right)^{\alpha-1} g(t) E_{\mu, \alpha, l}^{\gamma, \delta, c, c}\left(\omega t^{\mu} ; p\right) f(t) d t \mid \\
& \quad \leq \int_{a}^{m b}\left|\int_{a}^{t} g(s) E_{\mu, \alpha, l}^{\gamma, \delta, k, c}\left(\omega s^{\mu} ; p\right) d s\right|^{\alpha}\left|f^{\prime}(t)\right| d t \\
& \quad+\int_{a}^{m b}\left|\int_{t}^{m b} g(s) E_{\mu, \alpha, l}^{\gamma, \delta, k, c}\left(\omega s^{\mu} ; p\right) d s\right|^{\alpha}\left|f^{\prime}(t)\right| d t .
\end{aligned}
$$


Using absolute convergence of the Mittag-Leffler function and $\|g\|_{\infty}=\sup _{t \in[a, b]}|g(t)|$, we have

$$
\begin{aligned}
& \mid\left(\int_{a}^{m b} g(s) E_{\mu, \alpha, l}^{\gamma, \delta, k, c}\left(\omega s^{\mu} ; p\right) d s\right)^{\alpha}(f(a)+f(m b)) \\
& \quad-\alpha \int_{a}^{m b}\left(\int_{a}^{t} g(s) E_{\mu, \alpha, l}^{\gamma, \delta, k, c}\left(\omega s^{\mu} ; p\right) d s\right)^{\alpha-1} g(t) E_{\mu, \alpha, l}^{\gamma, \delta, c, c}\left(\omega t^{\mu} ; p\right) f(t) d t \\
& \quad-\alpha \int_{a}^{m b}\left(\int_{t}^{m b} g(s) E_{\mu, \alpha, l}^{\gamma, \delta, k, c}\left(\omega s^{\mu} ; p\right) d s\right)^{\alpha-1} g(t) E_{\mu, \alpha, l}^{\gamma, \delta, k, c}\left(\omega t^{\mu} ; p\right) f(t) d t \mid \\
& \quad \leq\|g\|_{\infty}^{\alpha} S^{\alpha}\left(\int_{a}^{m b}(t-a)^{\alpha}\left|f^{\prime}(t)\right| d t+\int_{a}^{m b}(m b-t)^{\alpha}\left|f^{\prime}(t)\right| d t\right) .
\end{aligned}
$$

Since $f^{\prime} \mid$ is an $m$-convex function, we have

$$
\left|f^{\prime}(t)\right| \leq \frac{m b-t}{m b-a}\left|f^{\prime}(a)\right|+m \frac{t-a}{m b-a}\left|f^{\prime}(b)\right|
$$

for $t \in[a, m b]$.

Using (14) in (13), we have

$$
\begin{aligned}
& \mid\left(\int_{a}^{m b} g(s) E_{\mu, \alpha, l}^{\gamma, \delta, k, c}\left(\omega s^{\mu} ; p\right) d s\right)^{\alpha}(f(a)+f(m b)) \\
& \quad-\alpha \int_{a}^{m b}\left(\int_{a}^{t} g(s) E_{\mu, \alpha, l}^{\gamma, \delta, k, c}\left(\omega s^{\mu} ; p\right) d s\right)^{\alpha-1} g(t) E_{\mu, \alpha, l}^{\gamma, \delta, c, c}\left(\omega t^{\mu} ; p\right) f(t) d t \\
& \quad-\alpha \int_{a}^{m b}\left(\int_{t}^{m b} g(s) E_{\mu, \alpha, l}^{\gamma, \delta, k, c}\left(\omega s^{\mu} ; p\right) d s\right)^{\alpha-1} g(t) E_{\mu, \alpha, l}^{\gamma, \delta, k, c}\left(\omega t^{\mu} ; p\right) f(t) d t \mid \\
& \leq\|g\|_{\infty}^{\alpha} S^{\alpha}\left(\int_{a}^{m b}(t-a)^{\alpha}\left(\frac{m b-t}{m b-a}\left|f^{\prime}(a)\right|+m \frac{t-a}{m b-a}\left|f^{\prime}(b)\right|\right) d t\right. \\
& \left.\quad+\int_{a}^{m b}(m b-t)^{\alpha}\left(\frac{m b-t}{m b-a}\left|f^{\prime}(a)\right|+m \frac{t-a}{m b-a}\left|f^{\prime}(b)\right|\right) d t\right) .
\end{aligned}
$$

After simple calculation of the above inequality, we get (11) which is required.

If we take $m=1$ in (11), then we get the following result for a convex function.

Corollary 2.4 Letf $:[a, b] \subseteq[0, \infty) \rightarrow \mathbb{R}$ be a differentiable function such that $f^{\prime} \in L_{1}[a, b]$ with $a<b$. Also let $g:[a, b] \rightarrow \mathbb{R}$ be a continuous function on $[a, b]$. If $\left|f^{\prime}\right|$ is a convex function on $[a, b]$, then the following inequality for extended generalized fractional integral operators holds:

$$
\begin{aligned}
& \mid\left(\int_{a}^{b} g(s) E_{\mu, \alpha, l}^{\gamma, \delta, k, c}\left(\omega s^{\mu} ; p\right) d s\right)^{\alpha}[f(a)+f(b)] \\
& \quad-\alpha \int_{a}^{b}\left(\int_{a}^{t} g(s) E_{\mu, \alpha, l}^{\gamma, \delta, k, c}\left(\omega s^{\mu} ; p\right) d s\right)^{\alpha-1} g(t) E_{\mu, \alpha, l}^{\gamma, \delta, k, c}\left(\omega t^{\mu} ; p\right) f(t) d t
\end{aligned}
$$




$$
\begin{aligned}
& -\alpha \int_{a}^{b}\left(\int_{t}^{b} g(s) E_{\mu, \alpha, l}^{\gamma, \delta, k, c}\left(\omega s^{\mu} ; p\right) d s\right)^{\alpha-1} g(t) E_{\mu, \alpha, l}^{\gamma, \delta, k, c}\left(\omega t^{\mu} ; p\right) f(t) d t \mid \\
\leq & \frac{(b-a)^{\alpha+1}\|g\|_{\infty}^{\alpha} S^{\alpha}}{(\alpha+1)}\left[\left|f^{\prime}(a)\right|+\left|f^{\prime}(b)\right|\right]
\end{aligned}
$$

for $k<\delta+\Re(\mu)$ and $\|g\|_{\infty}=\sup _{t \in[a, b]}|g(t)|$.

\section{Remark 2.5 In Theorem 2.3.}

(i) If we put $p=0$, then we get [6, Theorem 3.2].

(ii) If we put $\omega=p=0$ and $m=1$, then we get [13, Theorem 6].

(iii) If we take $\omega=p=0, m=1, \alpha=\frac{\mu}{k}$, and $g(s)=1$, then we get [8, Corollary 2.3].

(iv) For $g(s)=1$ along with $\omega=p=0, m=1$, and $\alpha=\mu$, we get [13, Corollary 2].

\section{Remark 2.6 In Corollary 2.4.}

(i) If we put $p=0$, then we get [1, Theorem 3.2].

(ii) If we put $\omega=p=0$, then we get [13, Theorem 6].

(iii) For $\omega=p=0, \alpha=\frac{\mu}{k}$, and $g(s)=1$, we get [8, Corollary 2.3].

(iv) For $g(s)=1$ along with $\omega=p=0$, we get [13, Corollary 2].

Next we give the following fractional integral inequality.

Theorem 2.7 Let $f:[a, m b] \rightarrow \mathbb{R}$ be a differentiable function such that $f \in L_{1}[a, m b]$ with $0 \leq a<m b$. Also let $g:[a, m b] \rightarrow \mathbb{R}$ be a continuous function on $[a, m b]$. If $\left|f^{\prime}\right|^{q}$ is a convex function on $[a, m b]$, then for $q>0$ the following inequality for extended generalized fractional integral operators holds:

$$
\begin{aligned}
& \mid\left(\int_{a}^{m b} g(s) E_{\mu, \alpha, l}^{\gamma, \delta, k, c}\left(\omega s^{\mu} ; p\right) d s\right)^{\alpha}(f(a)+f(m b)) \\
& \quad-\alpha \int_{a}^{m b}\left(\int_{a}^{t} g(s) E_{\mu, \alpha, l}^{\gamma, \delta, k, c}\left(\omega s^{\mu} ; p\right) d s\right)^{\alpha-1} g(t) E_{\mu, \alpha, l}^{\gamma, \delta, k, c}\left(\omega t^{\mu} ; p\right) f(t) d t \\
& \quad-\alpha \int_{a}^{m b}\left(\int_{t}^{m b} g(s) E_{\mu, \alpha, l}^{\gamma, \delta, k, c}\left(\omega s^{\mu} ; p\right) d s\right)^{\alpha-1} g(t) E_{\mu, \alpha, l}^{\gamma, \delta, k}\left(\omega t^{\mu} ; p\right) f(t) d t \mid \\
& \leq \frac{2(m b-a)^{\alpha+1}\|g\|_{\infty}^{\alpha} S^{\alpha}}{(\alpha p+1)^{\frac{1}{q}}}\left(\frac{\left|f^{\prime}(a)\right|^{q}+m\left|f^{\prime}(b)\right|^{q}}{2}\right)^{\frac{1}{q}}
\end{aligned}
$$

for $k<\delta+\Re(\mu)$ and $\|g\|_{\infty}=\sup _{t \in[a, m b]}|g(t)|$ and $\frac{1}{p}+\frac{1}{q}=1$.

Proof From Lemma 2.1 and by using Hölder's inequality, we have

$$
\begin{aligned}
& \mid\left(\int_{a}^{m b} g(s) E_{\mu, \alpha, l}^{\gamma, \delta, k, c}\left(\omega s^{\mu} ; p\right) d s\right)^{\alpha}(f(a)+f(m b)) \\
& \quad-\alpha \int_{a}^{m b}\left(\int_{a}^{t} g(s) E_{\mu, \alpha, l}^{\gamma, \delta, c,}\left(\omega s^{\mu} ; p\right) d s\right)^{\alpha-1} g(t) E_{\mu, \alpha, l}^{\gamma, \delta, k, c}\left(\omega t^{\mu} ; p\right) f(t) d t \\
& \quad-\alpha \int_{a}^{m b}\left(\int_{t}^{m b} g(s) E_{\mu, \alpha, l}^{\gamma, \delta, k, c}\left(\omega s^{\mu} ; p\right) d s\right)^{\alpha-1} g(t) E_{\mu, \alpha, l}^{\gamma, \delta, k, c}\left(\omega t^{\mu} ; p\right) f(t) d t \mid
\end{aligned}
$$




$$
\begin{aligned}
\leq & \left(\int_{a}^{m b}\left|\int_{a}^{t} g(s) E_{\mu, \alpha, l}^{\gamma, \delta, k, c}\left(\omega s^{\mu} ; p\right) d s\right|^{\alpha p} d t\right)^{\frac{1}{p}}\left(\int_{a}^{m b}\left|f^{\prime}(t)\right|^{q} d t\right)^{\frac{1}{q}} \\
& +\left(\int_{a}^{m b}\left|\int_{t}^{m b} g(s) E_{\mu, \alpha, l}^{\gamma, \delta, k, c}\left(\omega s^{\mu} ; p\right) d s\right|^{\alpha p} d t\right)^{\frac{1}{p}}\left(\int_{a}^{m b}\left|f^{\prime}(t)\right|^{q} d t\right)^{\frac{1}{q}} .
\end{aligned}
$$

Using absolute convergence of the Mittag-Leffler function and $\|g\|_{\infty}=\sup _{t \in[a, b]}|g(t)|$, we have

$$
\begin{aligned}
& \mid\left(\int_{a}^{m b} g(s) E_{\mu, \alpha, l}^{\gamma, \delta, k, c}\left(\omega s^{\mu} ; p\right) d s\right)^{\alpha}(f(a)+f(m b)) \\
& \quad-\alpha \int_{a}^{m b}\left(\int_{a}^{t} g(s) E_{\mu, \alpha, l}^{\gamma, \delta, c,}\left(\omega s^{\mu} ; p\right) d s\right)^{\alpha-1} g(t) E_{\mu, \alpha, l}^{\gamma, \delta, k}\left(\omega t^{\mu} ; p\right) f(t) d t \\
& \quad-\alpha \int_{a}^{m b}\left(\int_{t}^{m b} g(s) E_{\mu, \alpha, l}^{\gamma, \delta, k, c}\left(\omega s^{\mu} ; p\right) d s\right)^{\alpha-1} g(t) E_{\mu, \alpha, l}^{\gamma, \delta, k, c}\left(\omega t^{\mu} ; p\right) f(t) d t \mid \\
& \quad \leq\|g\|_{\infty}^{\alpha} S^{\alpha}\left(\left(\int_{a}^{m b}|t-a|^{\alpha p} d t\right)^{\frac{1}{p}}\right. \\
& \left.\quad+\left(\int_{a}^{m b}|m b-t|^{\alpha p} d t\right)^{\frac{1}{p}}\right)\left(\int_{a}^{m b}\left|f^{\prime}(t)\right|^{q} d t\right)^{\frac{1}{q}} .
\end{aligned}
$$

Since $\left|f^{\prime}(t)\right|^{q}$ is an $m$-convex function, we have

$$
\left|f^{\prime}(t)\right|^{q} \leq \frac{m b-t}{m b-a}\left|f^{\prime}(a)\right|^{q}+m \frac{t-a}{m b-a}\left|f^{\prime}(b)\right|^{q}
$$

Using (20) in (19), we have

$$
\begin{aligned}
& \mid\left(\int_{a}^{m b} g(s) E_{\mu, \alpha, l}^{\gamma, \delta, k, c}\left(\omega s^{\mu} ; p\right) d s\right)^{\alpha}(f(a)+f(m b)) \\
& \quad-\alpha \int_{a}^{m b}\left(\int_{a}^{t} g(s) E_{\mu, \alpha, l}^{\gamma, \delta, k, c}\left(\omega s^{\mu} ; p\right) d s\right)^{\alpha-1} g(t) E_{\mu, \alpha, l}^{\gamma, \delta, k, c}\left(\omega t^{\mu} ; p\right) f(t) d t \\
& \quad-\alpha \int_{a}^{m b}\left(\int_{t}^{m b} g(s) E_{\mu, \alpha, l}^{\gamma, \delta, k, c}\left(\omega s^{\mu} ; p\right) d s\right)^{\alpha-1} g(t) E_{\mu, \alpha, l}^{\gamma, \delta, k, c}\left(\omega t^{\mu} ; p\right) f(t) d t \mid \\
& \leq\|g\|_{\infty}^{\alpha} S^{\alpha}\left(\left(\int_{a}^{m b}|t-a|^{\alpha p} d t\right)^{\frac{1}{p}}+\left(\int_{a}^{m b}|m b-t|^{\alpha p} d t\right)^{\frac{1}{p}}\right) \\
& \quad \times\left(\int_{a}^{m b} \frac{m b-t}{m b-a}\left|f^{\prime}(a)\right|^{q}+m \frac{t-a}{m b-a}\left|f^{\prime}(b)\right|^{q}\right)^{\frac{1}{q}} .
\end{aligned}
$$

After simple calculation of the above inequality, we get (17) which is required.

If we take $m=1$ in (17), then we get the following result for a convex function.

Corollary 2.8 Let $f:[a, b] \subseteq[0, \infty) \rightarrow \mathbb{R}$ be a differentiable function such that $f^{\prime} \in L_{1}[a, b]$ with $a<b$. Also let $g:[a, b] \rightarrow \mathbb{R}$ be a continuous function on $[a, b]$. If $\left|f^{\prime}\right|^{q}$ is a convex function on $[a, b]$, then for $q>0$ the following inequality for extended generalized fractional 
integral operators holds:

$$
\begin{aligned}
& \mid\left(\int_{a}^{b} g(s) E_{\mu, \alpha, l}^{\gamma, \delta, k, c}\left(\omega s^{\mu} ; p\right) d s\right)^{\alpha}[f(a)+f(b)] \\
& \quad-\alpha \int_{a}^{b}\left(\int_{a}^{t} g(s) E_{\mu, \alpha, l}^{\gamma, \delta, k, c}\left(\omega s^{\mu} ; p\right) d s\right)^{\alpha-1} g(t) E_{\mu, \alpha, l}^{\gamma, \delta, k, c}\left(\omega t^{\mu} ; p\right) f(t) d t \\
& \quad-\alpha \int_{a}^{b}\left(\int_{t}^{b} g(s) E_{\mu, \alpha, l}^{\gamma, \delta, k, c}\left(\omega s^{\mu} ; p\right) d s\right)^{\alpha-1} g(t) E_{\mu, \alpha, l}^{\gamma, \delta, k, c}\left(\omega t^{\mu} ; p\right) f(t) d t \mid \\
& \quad \leq \frac{2(b-a)^{\alpha+1}\|g\|_{\infty}^{\alpha} S^{\alpha}}{(\alpha p+1)^{\frac{1}{q}}}\left[\frac{\left|f^{\prime}(a)\right|^{q}+\left|f^{\prime}(b)\right|^{q}}{2}\right]^{\frac{1}{q}}
\end{aligned}
$$

for $k<\delta+\Re(\mu)$ and $\|g\|_{\infty}=\sup _{t \in[a, b]}|g(t)|$ and $\frac{1}{p}+\frac{1}{q}=1$.

\section{Remark 2.9 In Theorem 2.7.}

(i) If we put $p=0$, then we get [6, Theorem 3.6].

(ii) If we put $\omega=p=0$ and $m=1$, then we get [13, Theorem 7].

(iii) If we take $\omega=p=0, m=1$ along with $\alpha=\frac{\mu}{k}$, then we get [8, Theorem 2.5].

(iv) If we take $g(s)=1, m=1$, and $\omega=p=0$, then we get [5, Theorem 2.3].

(v) If we put $\omega=p=0, m=1$, and $\alpha=1$, then we get [5, Corollary 3].

\section{Remark 2.10 In Corollary 2.8.}

(i) If we put $p=0$, then we get [1, Theorem 3.5].

(ii) If we put $\omega=p=0$, then we get [13, Theorem 7].

(iii) If we put $\omega=p=0, \alpha=1$, then we get [13, Corollary 3].

(iv) If we take $\omega=p=0$ along with $\alpha=\frac{\mu}{k}$, then we get [8, Theorem 2.5].

(v) If we take $g(s)=1$ and $\omega=p=0$, then we get [5, Theorem 2.3].

In the next result we give Hadamard type inequalities for $m$-convex functions via an extended Mittag-Leffler function.

Theorem 2.11 Let $f:[a, m b] \rightarrow \mathbb{R}$ be a function such that $\in L_{1}[a, m b]$ with $0 \leq a<m b$.If $f$ is $m$-convex on $[a, m b]$, then the following inequalities for extended generalized fractional integral operators hold:

$$
\begin{aligned}
2 f( & \left.\frac{a+m b}{2}\right) C_{\alpha,\left(\frac{a+m b}{2}\right)^{+}}(m b ; p) \\
\leq & \left(\epsilon_{\mu, \alpha, l, \omega^{\prime},\left(\frac{a+m b}{2}\right)^{+}}^{\gamma, \delta, k}\right)(m b ; p)+m^{\alpha+1}\left(\epsilon_{\mu, \alpha, l, m^{\mu} \omega^{\prime},\left(\frac{a+m b}{2 m}\right)}^{\gamma, \delta, k, c} f\right)\left(\frac{a}{m} ; p\right) \\
\leq & \frac{1}{m b-a}\left(f(a)-m^{2} f\left(\frac{a}{m^{2}}\right)\right) C_{\alpha+1,\left(\frac{a+m b}{2}\right)^{+}}(m b ; p) \\
& +m^{\alpha+1}\left(f(b)+m f\left(\frac{a}{m^{2}}\right)\right) C_{\alpha,\left(\frac{a+m b}{2 m}\right)^{-}}\left(\frac{a}{m} ; p\right),
\end{aligned}
$$

where $\omega^{\prime}=\frac{2^{\mu} \omega}{(m b-a)^{\mu}}$. 
Proof Since $f$ is an $m$-convex function, we have

$$
2 f\left(\frac{a+m b}{2}\right) \leq f\left(\frac{t}{2} a+\frac{2-t}{2} m b\right)+m f\left(\frac{2-t}{2 m} a+\frac{t}{2} b\right) .
$$

Also from $m$-convexity of $f$, we have

$$
\begin{aligned}
& f\left(\frac{t}{2} a+m \frac{2-t}{2} b\right)+m f\left(\frac{2-t}{2 m} a+\frac{t}{2} b\right) \\
& \quad \leq \frac{t}{2}\left(f(a)-m^{2} f\left(\frac{a}{m^{2}}\right)\right)+m\left(f(b)+m f\left(\frac{a}{m^{2}}\right)\right) .
\end{aligned}
$$

Multiplying (24) by $t^{\alpha-1} E_{\mu, \alpha, l}^{\gamma, \delta, c}\left(\omega t^{\mu} ; p\right)$ on both sides and then integrating over $[0,1]$, we have

$$
\begin{aligned}
2 f( & \left.\frac{a+m b}{2}\right) \int_{0}^{1} t^{\alpha-1} E_{\mu, \alpha, l}^{\gamma, \delta, k, c}\left(\omega t^{\mu} ; p\right) d t \\
\leq & \int_{0}^{1} t^{\alpha-1} E_{\mu, \alpha, l}^{\gamma, \delta, k}\left(\omega t^{\mu} ; p\right) f\left(\frac{t}{2} a+\frac{2-t}{2} m b\right) d t \\
& +m \int_{0}^{1} t^{\alpha-1} E_{\mu, \alpha, l}^{\gamma, \delta, k, c}\left(\omega t^{\mu} ; p\right) f\left(\frac{2-t}{2 m} a+\frac{t}{2} b\right) d t .
\end{aligned}
$$

Putting $u=\frac{t}{2} a+\frac{2-t}{2} m b$ and $v=\frac{2-t}{2 m} a+\frac{t}{2} b$ in (26), we have

$$
\begin{aligned}
& 2 f\left(\frac{a+m b}{2}\right) \int_{\frac{a+m b}{2}}^{m b}(m b-u)^{\alpha-1} E_{\mu, \alpha, l}^{\gamma, \delta, k, c}\left(\omega^{\prime}(m b-u)^{\mu} ; p\right) d u \\
& \quad \leq \int_{\frac{a+m b}{2}}^{m b}(m b-u)^{\alpha-1} E_{\mu, \alpha, l}^{\gamma, \delta, k, c}\left(\omega^{\prime}(m b-u)^{\mu} ; p\right) f(u) d u \\
& \quad+m^{\alpha+1} \int_{\frac{a}{m}}^{\frac{a+m b}{2 m}}\left(v-\frac{a}{m}\right)^{\alpha-1} E_{\mu, \alpha, l}^{\gamma, \delta, k, c}\left(m^{\mu} \omega^{\prime}\left(v-\frac{a}{m}\right)^{\mu}: p\right) f(v) d \nu .
\end{aligned}
$$

By using (3), (4), and (5) we get the first inequality of (23).

Now multiplying $(25)$ by $t^{\alpha-1} E_{\mu, \alpha, l}^{\gamma, \delta, k}\left(\omega t^{\mu} ; p\right)$ on both sides and then integrating over $[0,1]$, we have

$$
\begin{aligned}
\int_{0}^{1} t^{\alpha-1} E_{\mu, \alpha, l}^{\gamma, \delta, k, c}\left(\omega t^{\mu} ; p\right) f\left(\frac{t}{2} a+m \frac{2-t}{2} b\right) d t \\
\quad+m \int_{0}^{1} t^{\alpha-1} E_{\mu, \alpha, l}^{\gamma, \delta, k, c}\left(\omega t^{\mu} ; p\right) f\left(\frac{2-t}{2 m} a+\frac{t}{2} b\right) \\
\leq \frac{1}{2}\left(f(a)-m^{2} f\left(\frac{a}{m^{2}}\right)\right) \int_{0}^{1} t^{\alpha} E_{\mu, \alpha, l}^{\gamma, \delta, c}\left(\omega t^{\mu} ; p\right) d t \\
\quad+m\left(f(b)+m f\left(\frac{a}{m^{2}}\right)\right) \int_{0}^{1} t^{\alpha-1} E_{\mu, \alpha, l}^{\gamma, \delta, c}\left(\omega t^{\mu} ; p\right) d t .
\end{aligned}
$$


Putting $u=\frac{t}{2} a+m \frac{2-t}{2} b$ and $v=\frac{2-t}{2 m} a+\frac{t}{2} b$ in (27), we have

$$
\begin{aligned}
& \int_{\frac{a+m b}{2}}^{m b}(m b-u)^{\alpha-1} E_{\mu, \alpha, l}^{\gamma, \delta, k, c}\left(\omega^{\prime}(m b-u)^{\mu} ; p\right) f(u) d u \\
& \quad+\int_{\frac{a}{m}}^{\frac{a+m b}{2 m}}\left(v-\frac{a}{m}\right)^{\alpha-1} E_{\mu, \alpha, l}^{\gamma, \delta, k, c}\left(m^{\mu} \omega^{\prime}\left(v-\frac{a}{m}\right)^{\mu} ; p\right) f(v) d v \\
& \leq \frac{1}{2}\left(f(a)-m^{2} f\left(\frac{a}{m^{2}}\right)\right) \int_{\frac{a+m b}{2}}^{m b}(m b-u)^{\alpha} E_{\mu, \alpha, l}^{\gamma, \delta, k, c}\left(\omega^{\prime}(m b-u)^{\mu} ; p\right) d t \\
& \quad+m^{\alpha+1}\left(f(b)+m f\left(\frac{a}{m^{2}}\right)\right) \\
& \quad \times \int_{\frac{a}{m}}^{\frac{a+m b}{2 m}}\left(v-\frac{a}{m}\right)^{\alpha-1} E_{\mu, \alpha, l}^{\gamma, \delta, k, c}\left(m^{\mu} \omega^{\prime}\left(v-\frac{a}{m}\right)^{\mu} ; p\right) d t
\end{aligned}
$$

By using (3), (4), and (6), we get the second inequality of (23).

If we take $m=1$ in (23), then we get the following Hadamard type inequality for a convex function.

Corollary 2.12 Let $f:[a, b] \subseteq[0, \infty) \rightarrow \mathbb{R}$ be a function such that $f \in L_{1}[a, b]$ with $a<b$. Iff is convex on $[a, b]$, then the following inequalities for extended generalized fractional integral operators hold:

$$
\begin{aligned}
& f\left(\frac{a+b}{2}\right) C_{\alpha,\left(\frac{a+b}{2}\right)^{+}}(b ; p) \\
& \quad \leq\left[\left(\epsilon_{\mu, \alpha, l, \omega^{\prime},\left(\frac{a+b}{2}\right)^{+}}^{\gamma, \delta, k, c} f\right)(b ; p)+\left(\epsilon_{\mu, \alpha, l, \omega^{\prime},\left(\frac{a+b}{2}\right)_{-}^{\gamma}}^{\gamma, \delta, k),} f\right)(a ; p)\right] \\
& \quad \leq \frac{f(a)+f(b)}{2} C_{\alpha,\left(\frac{a+b}{2}\right)^{-}}(a ; p),
\end{aligned}
$$

where $\omega^{\prime}=\frac{2^{\mu} \omega}{(b-a)^{\mu}}$.

Remark 2.13 In Theorem 2.11.

(i) If we put $p=0$, then we get [6, Theorem 3.10].

(ii) If we put $\omega=p=0, m=1$, and $\alpha=1$, then we get the classical Hadamard inequality.

\section{Remark 2.14 In Corollary 2.12.}

(i) If we put $p=0$, then we get [1, Theorem 3.9].

(ii) If we put $\omega=p=0$ and $\alpha=1$, then we get the classical Hadamard inequality.

(iii) If we take $\omega=p=0$, then we get [14, Theorem 4].

\section{Concluding remarks}

We have investigated more general fractional integral inequalities. By selecting specific values of parameters quite interesting results can be obtained. For example selecting $p=0$, fractional integral inequalities for fractional integral operators defined by Salim and Faraj in [12], selecting $l=\delta=1$, fractional integral inequalities for fractional integral operators 
defined by Rahman et al. in [11], selecting $p=0$ and $l=\delta=1$, fractional integral inequalities for fractional integral operators defined by Shukla and Prajapati in [15] (see also [16]), selecting $p=0$ and $l=\delta=k=1$, fractional integral inequalities for fractional integral operators defined by Prabhakar in [10], selecting $p=\omega=0$, fractional integral inequalities for Riemann-Liouville fractional integral operators.

\section{Acknowledgements}

We thank the editor and referees for their careful reading and valuable suggestions to make the article reader friendly. The research work of Ghulam Farid is supported by COMSATS University Islamabad.

\section{Funding}

Not applicable.

\section{Competing interests}

It is declared that authors have no competing interests.

\section{Authors' contributions}

All authors have equal contribution in this article. All authors read and approved the final manuscript.

\section{Author details}

${ }^{1}$ Department of Mathematics, COMSATS University Islamabad, Attock, Pakistan. ${ }^{2}$ Department of Mathematics, University of Sargodha, Sargodha, Pakistan. ${ }^{3}$ General Studies Department, Jubail Industrial College, Jubail, Kingdom of Saudi Arabia ${ }^{4}$ GBPS Sherani, Hazro Attock, Pakistan.

\section{Publisher's Note}

Springer Nature remains neutral with regard to jurisdictional claims in published maps and institutional affiliations.

Received: 26 March 2018 Accepted: 28 August 2018 Published online: 15 September 2018

\section{References}

1. Abbas, G., Khan, K.A., Farid, G., Rehman, A.U.: Generalizations of some fractional integral inequalities via generalized Mittag-Leffler function. J. Inequal. Appl. 2017(1), 121 (2017)

2. Andrić, M., Farid, G., Pečarić, J.: A generalization of Mittag-Leffler function associated with Opial type inequalities due to Mitrinović and Pečarić. Submitted

3. Bakula, M.K., Ozdemir, M.E., Pečarić, J.: Hadamard type inequalities for m-convex and $(\alpha, m)$-convex functions. J. Inequal. Pure Appl. Math. 9(4), Article 9 (2008)

4. Dragomir, S.S.: On some new inequalities of Hermite-Hadamard type for m-convex functions. Tamkang J. Math. 33(1), 45-55 (2002)

5. Dragomir, S.S., Agarwal, R.P.: Two inequalities for differentiable mappings and applications to special means of real numbers and trapezoidal formula. Appl. Math. Lett. 11(5), 91-95 (1998)

6. Farid, G., Abbas, G.: Generalizations of some fractional integral inequalities for $m$-convex functions via generalized Mittag-Leffler function. Stud. Univ. Babeş-Bolyai, Math. 63(1), 23-35 (2018)

7. Farid, G., Marwan, M., Rehman, A.U.: New mean value theorems and generalization of Hadamard inequality via coordinated m-convex functions. J. Inequal. Appl. 2015(1), 283 (2015)

8. Farid, G., Rehman, A.U.: Generalizations of some integral inequalities for fractional integrals. Ann. Math. Sil. 32(1), 201-214 (2018)

9. Parmar, R.K.: A class of extended Mittag-Leffler functions and their properties related to integral transforms and fractional calculus. Mathematics 3, 1069-1082 (2015)

10. Prabhakar, T.R.: A singular integral equation with a generalized Mittag-Leffler function in the kernel. Yokohama Math. J. 19, 7-15 (1971)

11. Rahman, G., Baleanu, D., Qurashi, M.A., Purohit, S.D., Mubeen, S., Arshad, M.: The extended Mittag-Leffler function via fractional calculus. J. Nonlinear Sci. Appl. 10, 4244-4253 (2017)

12. Salim, T.O., Faraj, A.W.: A generalization of Mittag-Leffler function and integral operator associated with integral calculus. J. Fract. Calc. Appl. 3(5), 1-13 (2012)

13. Sarikaya, M.Z., Erden, S.: On the Hermite-Hadamard Fejér type integral inequality for convex functions. Turk. J. Anal. Number Theory 2(3), 85-89 (2014)

14. Sarikaya, M.Z., Yildirim, H.: On Hermite-Hadamard type inequalities for Riemann-Liouville fractional integrals. Miskolc Math. Notes 17(2), 1049-1059 (2017)

15. Shukla, A.K., Prajapati, J.C.: On a generalization of Mittag-Leffler function and its properties. J. Math. Anal. Appl. 336, 797-811 (2007)

16. Srivastava, H.M., Tomovski, Z:: Fractional calculus with an integral operator containing generalized Mittag-Leffler function in the kernel. Appl. Math. Comput. 211(1), 198-210 (2009)

17. Toader, G.H.: Some generalization of convexity. In: Proc. Colloq. Approx. Optim, Cluj Napoca (Romania), pp. 329-338 (1984)

18. Toader, G.H.: Some inequalities for m-convex functions. Stud. Univ. Babeş-Bolyai, Math. 38(1), 21-28 (1993)

19. Tomovski, Z., Hiller, R., Srivastava, H.M.: Fractional and operational calculus with generalized fractional derivative operators and Mittag-Leffler function. Integral Transforms Spec. Funct. 21(11), 797-814 (2011) 